\title{
Videoangiografía intraoperatoria con verde de indocianina durante la cirugía de aneurismas cerebrales. Experiencia inicial en 10 intervenciones quirúrgicas
}

\author{
E. Cordero; J. Enseñat; J. Macho*; J.J. González; M. Sánchez; C. Fernández; L. Caral; R. Valero** y E. Ferrer
}

Servicio de Neurocirugía. Servicio de Neuroangiorradiología*. Servicio de Anestesiología**. Hospital Clinic i Provincial de Barcelona.

Resumen

Objetivo. El objetivo de los autores es reportar nuestra experiencia inicial sobre la utilización de la videoangiografía intraoperatoria con verde de indocianina durante la cirugía de aneurismas cerebrales en nuestro centro.

Material y métodos. Se han realizado 10 procedimientos quirúrgicos en 9 pacientes, 5 varones y 4 mujeres entre 27 y 61 años con una media de edad de 49 años durante un tiempo de 10 meses entre marzo de 2008 y enero de 2009. Se realizaron 10 intervenciones y se cliparon 11 aneurismas, de los cuales 5 fueron diagnosticados tras su ruptura y 6 no. Se utilizaron verde de indocianina intravenosa y microscopio quirúrgico Leica $\mathrm{OH} 4$ con módulo de fluorescencia vascular intraoperatoria Leica FL800, con cámara infrarroja Sony (Heerbrugg-Suiza). Se recoge la información ofrecida por esta técnica durante la intervención y se compara con las imágenes de la arteriografía postoperatoria a las $\mathbf{2 4}$ horas. Se valoraron la oclusión total o parcial de la lesión aneurismática así como la preservación o no de la vascularización adyacente.

Resultados. Los hallazgos de la videoangiografía intraoperatoria sobre las lesiones aneurismáticas fueron de oclusión completa en todos los casos y permeabilidad de los vasos vecinos. Estos resultados se corresponden completamente con los de la arteriografía postoperatoria, excepto en un caso donde la arteriografía evidenció vasoespasmo moderado-grave sin repercusión clínica que durante la videoangiografía intraoperatoria no fue percibido. Clínicamente ningún paciente presentó déficits neurológicos añadidos.

Conclusiones. La videoangiografía intraoperatoria es una herramienta de fácil aplicación que ofrece información valiosa en cuanto a la oclusión completa del saco aneurismático y la permeabilidad de los vasos adyacentes a éste.

PALABRAS CLAVE: Videoangiografía intraoperatoria.

Recibido: 22-08-09. Aceptado: 13-10-09
Aneurismas cerebrales. Verde de indocianina. Microscopio quirúrgico.

Intraoperative videoangiography using green indocyanine during aneurysm surgery

Summary

Objective. The authors' objective is to report the initial appreciations on the use of the intraoperative nearinfrared indocyanine green videoangiography during aneurysm surgery in our center.

Method. 10 surgical procedures have been made in 9 patients, 5 males and 4 females between 27 and 61 years old with an average of age of 49 years during a time of 10 months between March, 2008 and January, 2009. 10 surgical procedures were performed and 11 aneurysms were clipped. Intravenous indocyanine green and surgical microscope Leica $\mathrm{OH} 4$ with module of vascular fluorescence intraoperating Leica FL800, with camera infrared Sony (Heerbrugg-Switzerland) were used. The information offered by this technique during the intervention is compared with the images of the postoperative angiography performed during the first 24 hours. The partial or complete occlusion and the respect to the near vessels were evaluated.

Results. The findings of the intraoperative videoangiography were the complete occlusion and absence of complications in all the cases. These results corresponded completely with the postoperative results of the angiography postoperative, except in a case where the angiography demonstrated vasoespasmo moderate without clinical repercussion that during the videoangiografía intraoperatoria was not perceived. Clinically no patient presented neurological added deficits.

Conclusions. The intraoperative videoangiography is a tool of easy application that offers valuable information as for the complete occlusion of the aneurysm and the permeability of the adjacent vessels.

KEY WORDS: Intraoperative videoangiography. Intracranial aneurysm. Indocyanine green. Surgical microscope. 


\section{Introducción}

El éxito de la cirugía de aneurismas cerebrales se basa en la correcta oclusión de la lesión y el mantenimiento del flujo en los vasos relacionados, sin embargo la arteriografía postoperatoria demuestra que existen entre 2 y $8 \%$ de clipajes incompletos y entre 4 y $12 \%$ de vasos adyacentes ocluidos $^{6}$. La utilización de la arteriografía intraoperatoria se ha propuesto como el método óptimo para corregir inmediatamente dichos errores, reportándose hasta un $30 \%$ de recambios intraoperatorios significativos del clip $^{1,3,5}$. Sin embargo las dificultades técnicas de su utilización limitan su aplicación en la mayoría de centros médicos. En los últimos años se han desarrollado nuevas opciones como la videoangiografía intraoperatoria como alternativas al método clásico.

Nuestro objetivo es hacer una valoración inicial de esta técnica en cuanto a su utilidad, eficacia y complicaciones.

\section{Material y métodos}

Se recogieron los datos de 10 procedimientos quirúrgicos de clipaje de 11 aneurismas en 9 pacientes diferentes. Cinco hombres y cuatro mujeres entre 27 y 61 años. La localización de los aneurismas intervenidos fue: 6 de arteria cerebral media izquierda, 1 de arteria cerebral media derecha, 1 de arteria carótida interna derecha y otro en espejo de arteria carótida interna izquierda, 1 de arteria comunicante anterior y 1 de coroidea anterior derecha. Cinco aneurismas fueron diagnosticados y tratados luego de su ruptura, cinco se diagnosticaron tras una hemorragia ocasionada por otro aneurisma y uno fue intervenido tras varios intentos fallidos de embolización. Tabla 1.

Se utilizó verde de indocianina como fármaco con propiedades fluorescentes y microscopio quirúrgico Leica $\mathrm{OH} 4$ con módulo de fluorescencia vascular intraoperatoria Leica FL800, con cámara infrarroja Sony.

La información valorada durante las intervenciones fue: la oclusión total o parcial de la lesión aneurismática y la preservación o no de la vascularización adyacente. Figura 1.

Dicha información fue comparada con los resultados de la arteriografía postoperatoria realizada dentro de las primeras 24 horas luego de la cirugía.

Se practicó un abordaje transilviano tras craneotomía pterional. En todos los casos se empleó como monitorización intraoperatoria potenciales evocados motores (PEM) de nervio mediano y peroneo mediante estimulación transcortical y somatosensitivos (PESS) de nervio mediano y poplíteo posterior. Además se registró la saturación yugular de oxígeno $\left(\mathrm{SjO}_{2}\right)$ y en los cinco casos de aneurismas rotos se valoró la presión tisular de oxígeno $\left(\mathrm{PtiO}_{2}\right)$ de las zonas cerebrales consideradas de riesgo durante la intervención, para lo cual se colocó el sensor en sustancia blanca luego de realizada la craneotomía; valores inferiores a $15 \mathrm{mmHg}$ fueron considerados indicadores de hipoxia tisular.

\section{Resultados}

Durante los 10 procedimientos quirúrgicos realizados se consideró, mediante la utilización de la videoangiografía intraoperatoria, que la oclusión fue completa y que la vascularización adyacente fue respetada en todos los casos.

Dichas apreciaciones se corresponden completamente con los resultados de la arteriografía postoperatoria. Sin embargo, y aunque el parámetro de vasoespasmo no fue tomado en cuenta como variable intraoperatoria, cabe mencionar que se evidenció vasoespasmo moderado sin

\section{Tabla 1}

\begin{tabular}{|l|c|c|c|c|c|c|c|c|c|c|c|}
\hline Aneurisma & 1 & $2 *$ & $3 *$ & 4 & 5 & 6 & 7 & 8 & $9 * *$ & $10^{* *}$ & 11 \\
\hline Sexo & $\mathrm{F}$ & $\mathrm{M}$ & $\mathrm{M}$ & $\mathrm{M}$ & $\mathrm{M}$ & $\mathrm{F}$ & $\mathrm{M}$ & $\mathrm{F}$ & $\mathrm{M}$ & $\mathrm{M}$ & $\mathrm{M}$ \\
\hline Edad & 59 & 53 & 53 & 46 & 27 & 61 & 47 & 54 & 52 & 52 & 44 \\
\hline Hunt y Hess & $\mathrm{I}$ & $\mathrm{III}$ & & & & $\mathrm{II}$ & & $\mathrm{II}$ & & & $\mathrm{I}$ \\
\hline Fisher & 2 & 3 & & & & 3 & & 2 & & & 1 \\
\hline Localización & ACMI & ACD & ACI & ACMI & Cor.A. & ACMI & Co.A. & ACMD & ACMI & ACMI & ACMI \\
\hline
\end{tabular}

*Aneurismas en espejo tratados en dos tiempos quirúrgicos. **2 aneurismas de arteria cerebral media izquierda del mismo paciente intervenidos durante el mismo procedimiento quirúrgico. ACMI: arteria cerebral media izquierda. ACD: arteria carótida derecha. ACI: arteria carótida izquierda. ACMI: arteria cerebral media izquierda. Cor.A.: arteria coroidea anterior. CO.A.: arteria comunicante anterior. 


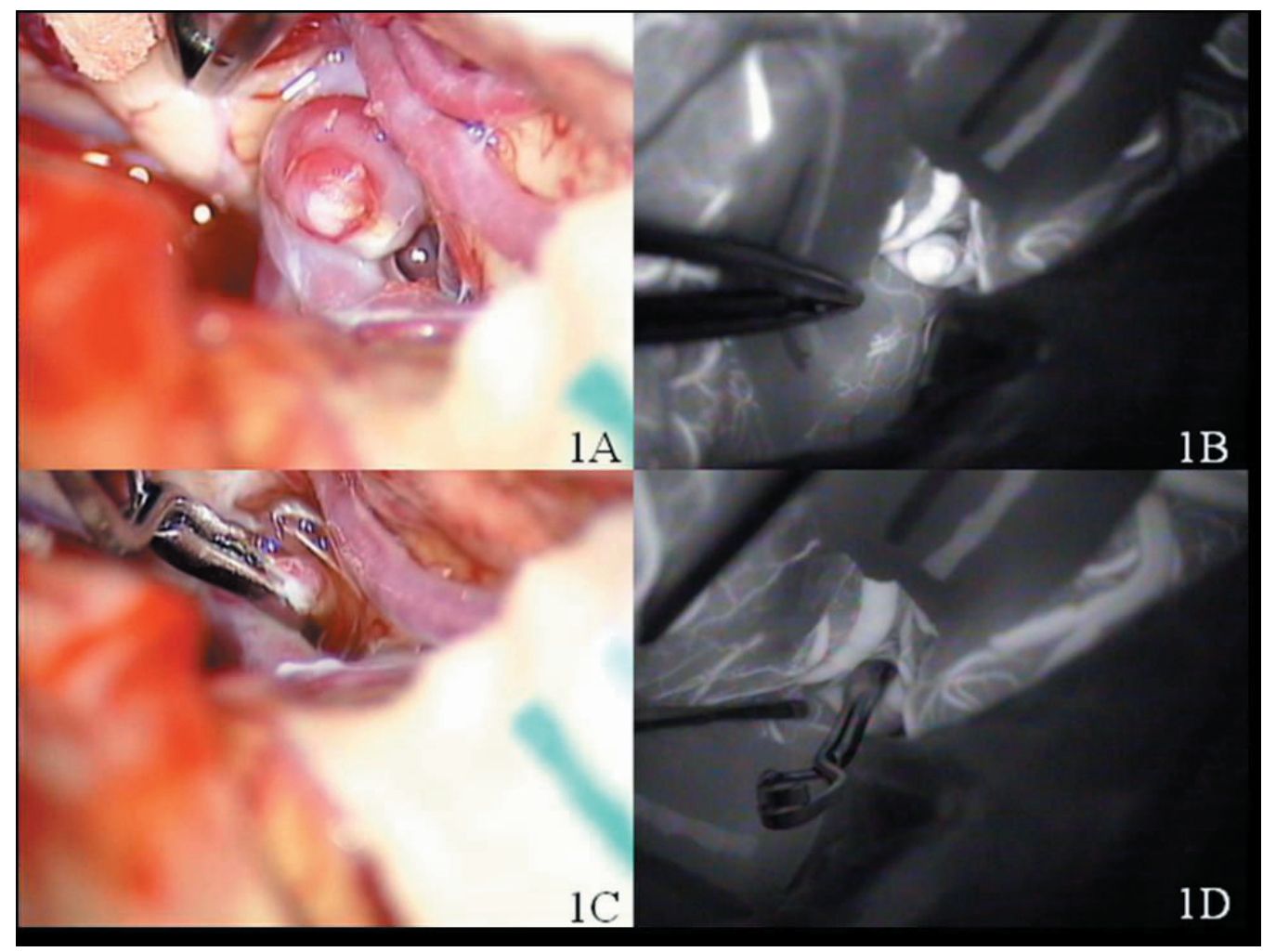

Figura 1. 1A Imagen intraoperatoria de aneurisma de arteria cerebral media antes del clipaje. $1 B$ Imagen fluorescente intraoperatoria de aneurisma de arteria cerebral media antes del clipaje. $1 C$ Imagen intraoperatoria de aneurisma de arteria cerebral media después del clipaje. $1 D$ Imagen fluorescente intraoperatoria de aneurisma de arteria cerebral media luego del clipaje.

repercusión clínica en uno de los casos de aneurisma de arteria cerebral media izquierda durante la arteriografía postoperatoria que durante la videoangiografía intraoperatoria no fue percibido.

Luego de los procedimientos no se evidenció morbilidad añadida en ninguno de los casos, existiendo concordancia completa con la monitorización intraoperatoria. Tampoco se apreciaron reacciones adversas al fármaco empleado.

\section{Discusión}

La utilización de sustancias con propiedades fluorescentes como el verde de indocianina es una práctica habitual en la medicina moderna. Dicho fármaco ha sido ampliamente empleado en pruebas funcionales hepáticas y en la Oftalmología. Actualmente se ha iniciado su utilización en la Neurocirugía.

Se trata de una sustancia con escasos efectos colaterales reportados, salvo reacciones alérgicas generalmente leves. Se aplica por vía intravenosa y es rápidamente eliminada (menos de 10 minutos) por vía hepática en un $99 \%$. Se difunde por toda la circulación corporal de forma casi inmediata. La dosis recomendada es de $0,2-0,5 \mathrm{mg} / \mathrm{kg}$ peso. Dosis habitual $25 \mathrm{mg}^{6}$.

La aplicación de rayos infrarrojos genera un aumento en la longitud de onda de la sustancia (fluorescencia) que es captada por una cámara adaptada incorporada al microscopio quirúrgico ${ }^{7}$.
Dentro de los estudios realizados destaca el de A. Raabe, et.al ${ }^{6}$, en el que se compara de forma prospectiva la videoangiografía intraoperatoria con la arteriografía. En dicho estudio se empleó verde de indocianina y un microscopio quirúrgico diferente al del presente trabajo. Sus resultados en 187 procedimientos quirúrgicos en 114 pacientes revelaron una concordancia del $90 \%$ con los de la arteriografía. Solamente en tres casos se evidenciaron hallazgos relevantes durante la arteriografía que no fueron visualizados durante la videoangiografía intraoperatoria y sólo uno de ellos requirió una nueva intervención quirúrgica.

Otros estudios realizados demuestran la utilidad de la técnica en la cirugía de malformaciones cerebrales arteriovenosas, el by-pass extra-intracraneal y el estudio intraoperatorio de las arteria perforantes durante la cirugía aneurismática ${ }^{4,8,9}$. Se trata de series de casos clínicos donde se menciona las aplicaciones de la técnica durante dichos procedimientos neuroquirúrgicos.

La técnica de videoangiografía intraoperatoria permite al cirujano realizar una valoración intraoperatoria de la vascularización cerebral de una manera dinámica antes, durante y después de la colocación del clip, lo cual admite la posibilidad de tomar decisiones de forma inmediata sin la necesidad de retirar la mirada del campo quirúrgico. La arteriografía intraoperatoria requiere un tiempo cercano a los 20 minutos $^{2}$ para obtener imágenes de la circulación cerebral; este tiempo puede ser vital en caso de que el clip 
deba ser retirado por oclusión de vasos adyacentes.

Creemos que el principal inconveniente de la videoangiografía intraoperatoria es que sólo nos permite ver la imagen del campo quirúrgico, siendo imprescindible la disección del todo el aneurisma y los vasos adyacentes. Cualquier vaso o parte del aneurisma que quede oculto por su dirección o bien por sangre no podrá ser valorado por ésta. La ventaja de la arteriografía es que la capacidad de sustracción y las diferentes proyecciones nos permiten una apreciación de todo el árbol vascular cerebral.

\section{Conclusiones}

Consideramos que la videoangiografía intraoperatoria es una técnica dinámica, inocua, no invasiva, de fácil y rápida aplicación que ofrece resultados similares a los de la arteriografía intraoperatoria durante la cirugía de aneurismas cerebrales. Nuestros resultados son concordantes con los descritos por la literatura si bien es necesario un número mayor de pacientes.

\section{Bibliografía}

1. Alexander, T.D., Macdonald, R.L., Weir, B., Kowalczuk, A.: Intraoperative angiography in cerebral aneurysm surgery: a prospective study of 100 craniotomies. Neurosurgery 1996; 39: 10-18.

2. Batjer, H.H., Frankfurt, A.I., Purdy, P.D., Smith, S.S., Samson, D.S.: Use of etomidate, temporary arterial occlusion, and intraoperative angiography in surgical treatment of large and giant cerebral aneurysms. J Neurosurg 1988; 68: 234240.

3. Chiang,V.L., Gailloud, P., Murphy, K.J., Rigamonti, D., Tamargo, R.J.: Routine intraoperative angiography during aneurysm surgery. J Neurosurg 2002; 96: 979-980.

4. De Oliveira, J.G., Beck, J., Seifert, V., Texeira, M.J.,
Raabe A.: Assessment of flow in perforating arteries during intracranial aneurysm surgery using intraoperative near-infrared indocyanine green videoangiography. Neurosurgery 2007; 61: ONS-63-ONS-73.

5. Payner, T. D., Horner, T.G., Leipzig, T.J., Scott, J.A., Gilmor, R.L., DeNardo, A. J.: Role of intraoperative angiography in the surgical treatment of cerebral aneurysms. J Neurosurg 1998; 88: 441-448.

6. Raabe, A., Nakaji, P., Beck J., et al.: Prospective evaluation of surgical microscope-integrated intraoperative near-infrared indocyanine green videoangiography during aneurysm surgery. J Neurosurg 2005; 103: 982-989.

7. Raabe, A., Beck, J., Seifert, V.: Technique and image quality of intraoperative indocyanine green angiography during aneurysm surgery using surgical microscope integrated near-infrared video technology. Zentralbl Neurochir 2005; 66: $1-6$.

8. Takagi, Y., Kikuta, K., Nozaki, K., Sawamura, K., Hashimoto, N.: Detection of a residual nidus by surgical microscope-integrated intraoperative near-infrared indocyanine green videoangiography in a child with a cerebral arteriovenous malformation. J Neurosurg Ped 2007; 157.

9. Woitzik, J., Horn, P., Vajkoczy, P., Schmiedek, P.: Intraoperative control of extracranial-intracranial bypass patency by near-infrared indocyanine green videoangiography. J Neuro$\operatorname{surg} 2005$; 102: 692-698.

Cordero, E.; Enseñat, J.; Macho, J.; González, J.; Sánchez, M.; Fernández, C.; Caral, L.; Valero, R.; Ferrer, E.: Videoangiografía intraoperatoria con verde de indocianina durante la cirugía de aneurismas cerebrales. Experiencia inicial en 10 intervenciones quirúrgicas. Neurocirugía 2010; 21: 302-305.

Correspondencia: Esteban Cordero. Calle Rubio i Ors 1-3 $4^{\mathrm{a}}$ Planta $3^{\text {a }}$ Puerta. 08940 Cornella de Llobregat. Barcelona. 